\title{
Subband Approach to Bandlimited Crosstalk Cancellation System in Spatial Sound Reproduction
}

\author{
Mingsian R. Bai and Chih-Chung Lee \\ Department of Mechanical Engineering, National Chiao-Tung University, 1001 Ta-Hsueh Road, Hsin-Chu 300, Taiwan
}

Received 27 December 2005; Revised 1 May 2006; Accepted 16 July 2006

Recommended by Yuan-Pei Lin

Crosstalk cancellation system (CCS) plays a vital role in spatial sound reproduction using multichannel loudspeakers. However, this technique is still not of full-blown use in practical applications due to heavy computation loading. To reduce the computation loading, a bandlimited CCS is presented in this paper on the basis of subband filtering approach. A pseudoquadrature mirror filter (QMF) bank is employed in the implementation of CCS filters which are bandlimited to $6 \mathrm{kHz}$, where human's localization is the most sensitive. In addition, a frequency-dependent regularization scheme is adopted in designing the CCS inverse filters. To justify the proposed system, subjective listening experiments were undertaken in an anechoic room. The experiments include two parts: the source localization test and the sound quality test. Analysis of variance (ANOVA) is applied to process the data and assess statistical significance of subjective experiments. The results indicate that the bandlimited CCS performed comparably well as the fullband CCS, whereas the computation loading was reduced by approximately eighty percent.

Copyright (c) 2007 M. R. Bai and C.-C. Lee. This is an open access article distributed under the Creative Commons Attribution License, which permits unrestricted use, distribution, and reproduction in any medium, provided the original work is properly cited.

\section{INTRODUCTION}

The fundamental idea of spatial audio reproduction is to synthesize a virtual sound image so that the listener perceives as if the signals reproduced at the listener's ears would have been produced by a specific source located at an intended position relative to the listener $[1,2]$. This attractive feature of spatial audio lends itself to an emerging audio technology with promising application in mobile phone, personal computer multimedia, video games, home theater, and so forth.

The rendering of spatial audio is either by headphones or by loudspeakers. Headphones reproduction is straightforward, but suffers from several shortcomings such as in-head localization, front-back reversal, and discomfort to wear. While loudspeakers do not have the same problems as the headphones, another issue adversely affects the performance of spatial audio rendering using loudspeakers. The issue associated with loudspeakers is the crosstalks at the contralateral paths from the loudspeakers to the listener's ears that may obscure the sense of source localization due to the Haas effect [3]. To overcome the problem, crosstalk cancellation systems (CCS) that seek to minimize, if not totally eliminate, crosstalk have been studied extensively by researchers [4-9]. Methods of designing CCS are divided into two kinds of approaches including time domain and frequency domain. Kirkeby and Nelson proposed an LS time-domain filtering to approximate the desired inverse function [10]. In contrast to the time-domain method that is time consuming for long filters, a fast frequency-domain deconvolution method offers more advantage in terms of computational speed [11].

Notwithstanding the preliminary success of CCS in academic community, two problems seriously hamper the use of CCS in practical applications. One stems from the limited size of the so-called "sweet spot" in which CCS remains effective. The sweet spots are generally so small especially at lateral side that a head movement of a few centimeters would completely destroy the cancellation performance. Two kinds of approaches can be used to address this problem-the adaptive design and the robust design. An example of adaptive CCS with head tracker was presented in the work of Kyriakakis et al. [12], and Kyriakakis [13]. This approach dynamically adjusts the CCS filters by tracking the head position of the listener using optical or acoustical sensors. However, the approach has not been widely used because of the increased hardware and software complexity of the head tracker. On the other hand, instead of dynamically tracking the listener's head, an alternative CCS design using fixed filters can be taken to create a "wide" sweet spot that accommodates larger 
head movement. A well-known example of robust CCS is "stereo dipole" presented by Kirkeby et al. [14]. Other approaches with multidrive loudspeakers have been suggested by Bai et al. [15], Takeuchi et al. [16], and Yang et al. [17, 18]. The other problem is computation loading due to multichannel filtering and long-length filters. In general, finer frequency resolution, that is, long impulse response, is needed for excellent reproduction, especially in a reverberated room.

The emphasis of this paper is placed on reducing computation loading. In considering the robustness against uncertainties of HRTFs (head-related transfer function) and head movement and head shadowing effect at high frequencies, the proposed CCS is bandlimited to frequencies below $6 \mathrm{kHz}$ [19]. That is, the CCS only functions at low frequencies and the binaural signals are directly passed through at high frequencies. The bandlimited implementation approach suggested in [19] is more computationally demanding due to its fixed operating rate. In this work, we adopted a subband filtering technique based on a cosine modulated quadrature mirror filter (QMF) bank [20]. In this design, the approximated perfect reconstruction condition is fulfilled and the CCS is operated at low rate. Therefore, it can use more effort at low frequencies for characteristics of human perceptual hearing. Another feature of the proposed system is that CCS filter is designed with frequency-dependent regularization [21]. The present approach which differs itself from the methods using constant regularization [11] provides more flexibility in the design stage. In order to verify the proposed CCS, subjective listening experiments were conducted to compare it to the traditional CCS. The results of subjective tests will be validated by using analysis of variance (ANOVA). The intention is to develop the CCS with light computation loading that performs comparably well as the fullband CCS.

\section{MULTICHANNEL INVERSE FILTERING FOR CCS FROM A MODEL-MATCHING PERSPECTIVE}

The CCS aims to cancel the crosstalks in the contralateral paths from the stereo loudspeakers to the listener's ears so that the binaural signals are reproduced at two ears like those reproduced using a headphone. This problem can be viewed from a model-matching perspective, as shown in Figure 1. In the block diagram, $\mathbf{x}(z)$ is a vector of $Q$ program input signals, $\mathbf{v}(z)$ is a vector of $P$ loudspeaker input signals, and $\mathbf{e}(z)$ is a vector of $L$ error signals. $\mathbf{M}(z)$ is an $L \times Q$ matrix of matching model, $\mathbf{H}(z)$ is an $L \times P$ plant transfer matrix, and $\mathrm{C}(z)$ is a $P \times Q$ matrix of the CCS filters. The $z^{-m}$ term accounts for the modeling delay to ensure causality of the CCS filters. Let us neglect the modeling delay for the moment; it is straightforward to write down the input-output relationship:

$$
\mathbf{e}(z)=[\mathbf{M}(z)-\mathbf{H}(z) \mathbf{C}(z)] \mathbf{x}(z)
$$

For arbitrary inputs, minimization of the error output is tantamount to the following optimization problem:

$$
\min _{\mathbf{C}}\|\mathbf{M}-\mathbf{H C}\|_{F}^{2}
$$

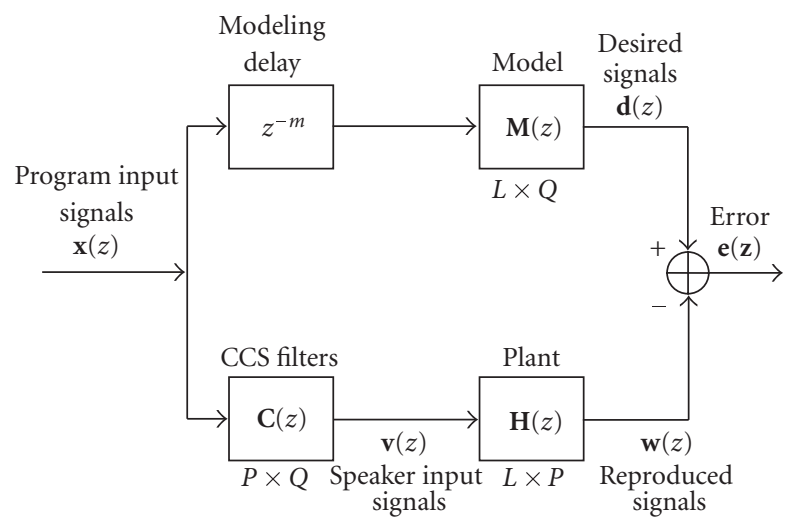

FIGURE 1: The block diagram of a multichannel model-matching problem in the CCS design.

where $F$ symbolizes the Frobenius norm [22]. For an $L \times Q$ matrix A, Frobenius norm is defined as

$$
\begin{aligned}
\|\mathbf{A}\|_{F}^{2} & =\sum_{q=1}^{Q} \sum_{l=1}^{L}\left|a_{l q}\right|^{2} \\
& =\sum_{q=1}^{Q}\left\|\mathbf{a}_{q}\right\|_{2}^{2}, \quad \mathbf{a}_{q} \text { being the } q \text { th column of } \mathbf{A} .
\end{aligned}
$$

Hence, the minimization problem of Frobenius norm can be converted to the minimization problem of 2-norm by partitioning the matrices into columns. Specifically, since there is no coupling between the columns of the matrix $\mathbf{C}$, the minimization of the square of the Frobenius norm of the entire matrix $\mathbf{H}$ is tantamount to minimizing the square of each column independently. Therefore, (2) can be rewritten into

$$
\min _{\mathbf{c}_{q}, \min _{q=1,2, \ldots, Q}} \sum_{q=1}^{Q}\left\|\mathbf{H c}_{q}-\mathbf{m}_{q}\right\|_{2}^{2},
$$

where $\mathbf{c}_{q}$ and $\mathbf{m}_{q}$ are the $q$ th column of the matrices $\mathbf{C}$ and $\mathbf{M}$, respectively. The optimal solution of $\mathbf{c}_{q}$ can be obtained by applying the method of least squares to each column:

$$
\mathbf{c}_{q}=\mathbf{H}^{+} \mathbf{m}_{q}, \quad q=1,2, \ldots, Q,
$$

where $\mathbf{H}^{+}$is the pseudoinverse of $\mathbf{H}$ [22]. This optimal solution in the least-square sense can be assembled in a more compact matrix form:

$$
\left[\begin{array}{llll}
\mathbf{c}_{1} & \mathbf{c}_{2} & \cdots & \mathbf{c}_{Q}
\end{array}\right]=\mathbf{H}^{+}\left[\begin{array}{llll}
\mathbf{m}_{1} & \mathbf{m}_{2} & \cdots & \mathbf{m}_{Q}
\end{array}\right]
$$

or

$$
\mathbf{C}=\mathbf{H}^{+} \mathbf{M} \text {. }
$$

For a matrix $\mathbf{H}$ with full-column $\operatorname{rank}(L \geq P), \mathbf{H}^{+}$can be calculated according to

$$
\mathbf{H}^{+}=\left(\mathbf{H}^{H} \mathbf{H}\right)^{-1} \mathbf{H}^{H} .
$$


Here, $\mathbf{H}^{+}$is also referred to as the left-pseudoinverse of $\mathbf{H}$ such that $\mathbf{H}^{+} \mathbf{H}=\mathbf{I}$.

In practice, the number of loudspeakers is usually greater than the number of ears, that is, $L \leq P$. Regularization can be used to prevent the singularity of $\mathbf{H}^{H} \mathbf{H}$ from saturating the filter gains $[11,23]$ :

$$
\mathbf{H}^{+}=\left(\mathbf{H}^{H} \mathbf{H}+\gamma \mathbf{I}\right)^{-1} \mathbf{H}^{H} .
$$

The regularization parameter $\gamma$ can either be constant or frequency-dependent [21]. A frequency-dependent $\gamma$ is based on a gain threshold on the maximum of the absolute values of all entries in $\mathbf{C}$. If the threshold is exceeded, a larger $\gamma$ should be chosen. The binary search method can be used to accelerate the search. It is noted that the procedure to obtain the filter $\mathbf{C}$ in (6) is essentially a frequency-domain formulation; inverse Fourier transform along with circular shift (hence the modeling delay) is needed to obtain causal FIR (finite impulse response) filters.

\section{BANDLIMITED IMPLEMENTATION USING THE MULTIRATE APPROACH}

Bandlimited implementation is chosen in this work for several reasons. First, the computation loading is too high to afford a fullband $(0 \sim 20 \mathrm{kHz})$ implementation. For the example of the stereo loudspeaker considered herein, the CCS would contain 4 filters. If each filter has 3000 taps, the convolution would require $1.2 \times 10^{4}$ multiplications and additions per sample interval. Except for special-purpose DSP engine, real time implementation for a fullband CCS is usually prohibitive for the sampling rate commonly used in audio processing, for example, $44.1 \mathrm{kHz}$ or $48 \mathrm{kHz}$. Second, at high frequencies, the wavelength could be much smaller than a head width. Under this circumstance, the CCS would be extremely susceptible to misalignment of the listener's head and uncertainties involved in HRTF modeling. Third, at high frequencies, a listener's head provides natural shadowing for the contralateral paths, which is more robust than direct application of CCS. The CCS in this study is chosen to be bandlimited to $6 \mathrm{kHz}$ (the wavelength at this frequency is approximately $5.6 \mathrm{~cm}$ ). To accomplish this, a 4-channel pseudo-QMF bank is employed to divide the total audible frequency range into subbands for CCS and direct transmission, respectively.

The design strategy of subband filter bank employed in this paper is the cosine modulated pseudo-QMF. In this method, a FIR filter must be selected as the prototype. Using this prototype, an $M$-channel maximally decimated filter bank (number of subbands = up/down sampling factor) is generated with the aid of cosine modulation. The maximum attenuation that can be attained by a perfectly reconstructing $(\mathrm{PR})$ cosine modulated filter bank is about $40 \mathrm{~dB}$. Nevertheless, this PR filter bank would still present an undesirable ringing problem. To alleviate this problem, the PR condition is relaxed in the FIR filter design to gain more stopband attenuation. From our experience, as much as $60 \mathrm{~dB}$ attenuation is required for acceptable reproduction.

Based on the method in [20], the following analysis and synthesis filter banks represented by $g_{k}(z)$ and $f_{k}(z)$, respec- tively, are employed to minimize phase distortion and aliasing:

$$
\begin{gathered}
g_{k}(n)=2 p_{0}(n) \cos \left(\frac{\pi}{M}(k+0.5)\left(n-\frac{N}{2}\right)+\theta_{k}\right), \\
f_{k}(n)=g_{k}(N-n),
\end{gathered}
$$

where $\theta_{k}=(-1)^{k}(\pi / 4), 0 \leq k \leq M-1$, and $p_{0}(n), n=$ $1,2, \ldots, N$ are the coefficients of the prototype FIR filter. The remaining problem is how to minimize the amplitude distortion. The distortion function $T(z)$ for the filter bank is given as in [20]:

$$
T(z)=\frac{1}{M} \sum_{k=0}^{M-1} F_{k}(z) G_{k}(z) .
$$

$Z$-transform of (10) leads to $F_{k}(z)=z^{-N} \widetilde{G}_{k}(z)$, where $\widetilde{G}_{k}(z)$ is the paraconjugation of $G_{k}(z)$. The distortion function can thus be written in frequency domain as

$$
T\left(e^{j \omega}\right)=\frac{1}{M} e^{-j \omega N} \sum_{k=0}^{M-1}\left|G_{k}\left(e^{j \omega}\right)\right|^{2} .
$$

A filter $P(z)$ is called a Nyquist $(M)$ filter if the following condition is met:

$$
p(M n)= \begin{cases}c, & n=0 \\ 0, & \text { otherwise }\end{cases}
$$

where $p(n)$ is the impulse response of $P(z)$ and $c$ is a constant. In frequency domain,

$$
\sum_{k=0}^{M-1} P\left(e^{j(\omega-2 \pi k / N)}\right)=M c
$$

Equations (12) and (14) indicate that if $\left|G_{k}\left(e^{j \omega}\right)\right|^{2}$ is a Nyquist $(M)$ filter, or equivalently $\left|P_{0}\left(e^{j \omega}\right)\right|^{2}$ is a Nyquist (2M) filter, the magnitude of $T(z)$ will be flat.

In this QMF design, the Kaiser window is used as the FIR prototype [24]. Given the specifications of transition bandwidth $\Delta f$ and stopband attenuation $A_{s}$, the parameter $\beta$ and the filter order $N$ can be determined according to

$$
\begin{gathered}
\beta= \begin{cases}0.1102\left(A_{s}-8.7\right) & \text { if } A_{s}>50, \\
0.5842\left(A_{s}-21\right)^{0.4}+0.07886\left(A_{s}-21\right) & \text { if } 21<A_{s}<50 \\
0 & \text { if } A_{s}<21,\end{cases} \\
N=\frac{A_{s}-7.95}{14.36 \Delta f} .
\end{gathered}
$$

An optimization procedure is employed here to make $P_{0}(z) \widetilde{P}_{0}(z)$ an approximate Nyquist $(2 M)$ filter, as posed by the following min-max problem [24]:

$$
\min _{\omega_{c}} \max _{n \neq 0}\left|p_{0}(n) * p_{0}(-n)\right|_{\downarrow 2 M}
$$


where the asterisk $*$ denotes the convolution operator. Because this is a convex problem, optimal cutoff frequency can always be found [24]. After obtaining the optimal prototype filter, the analysis and synthesis filters are generated according to (9) and (10), respectively. The filter bank can be easily implemented with techniques such as polyphase structure or discrete cosine transform (DCT) [20].

\section{SUBJECTIVE EXPERIMENTS}

In order to compare the performance of the proposed CCS and the fullband CCS, subjective experiments were undertaken in an anechoic room. The experimental arrangement is shown in Figure 2. This experiment employed a stereophonic two-way loudspeaker system, ELAC BS 103.2. The microphone and the preamplifier are GRAS 40AC and GRAS 26AM, respectively. The plant transfer function matrices were measured on an acoustical manikin, KEMAR (Knowles electronics manikin for acoustic research), along with the ear model, DB-065. The frequency responses of the plants are shown in Figure 3 wherein the solid line and dotted line represent the ipsilateral and the contralateral paths, respectively. Only responses measured on the right ear are shown because of the assumed symmetry. The $x$-axis is logarithmic frequency in $\mathrm{Hz}$ and the $y$-axis is magnitude in $\mathrm{dB}$. The CCS filters with 3000 taps are designed according to the method presented in Section 2 with $12 \mathrm{~dB}$ threshold. The matrix $\mathbf{Q}$ is defined as

$$
\mathbf{Q}=\left[\begin{array}{ll}
Q_{11} & Q_{12} \\
Q_{21} & Q_{22}
\end{array}\right]=\mathbf{H C} .
$$

This matrix attempts to approximate the model matrix $\mathbf{M}$ which is set to be an identity matrix here. Figure 4(a) shows the frequency responses of $Q_{11 f}$ and $Q_{12 f}$, where the subscript $f$ stands for the fullband method, represented as solid line and dotted line, respectively. After compensation, the ipsilateral magnitude is almost flat from $300 \mathrm{~Hz}$ to $8 \mathrm{kHz}$. Some imperfect match can be seen at low frequencies and at high frequencies because the CCS filter gain is constrained, that is, large regularization. On the other hand, the contralateral magnitude is degraded to around $-40 \mathrm{~dB}$. Channel separation, defined as the ratio of the contralateral response and the ipsilateral response, is employed as a performance index. The channel separation, $Q_{12 f} / Q_{11 f}$, is shown in Figure 4(b) as the dotted line. The solid line represents the natural channel separation, $H_{12} / H_{11}$. As mentioned above, the fullband approach is impractical due to many reasons. The proposed method in this work is bandlimited to $6 \mathrm{kHz}$ with $48 \mathrm{kHz}$ sampling rate. The block diagram of the bandlimited CCS is illustrated in Figure 5. Through the use of the method presented in Section 3, the prototype FIR filter with 120 taps and the analysis bank are plotted in Figures 6(a) and 6(b), respectively. The CCS only functions at the lowest band and operates at lower sampling rate. The computation load of an analysis bank or a synthesis bank equals to that of the prototype FIR filter when the polyphase structure is employed. Since CCS operates at low rate, it is able to sample more frequencies at design stage. In the experiment, the tap of the

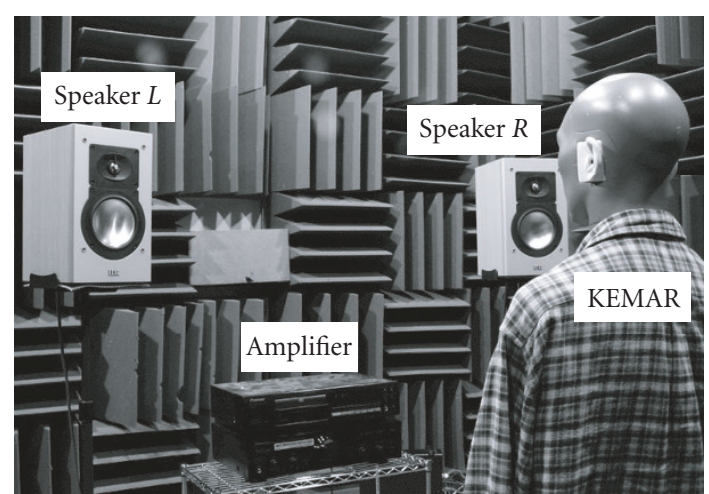

Figure 2: The experimental configuration.

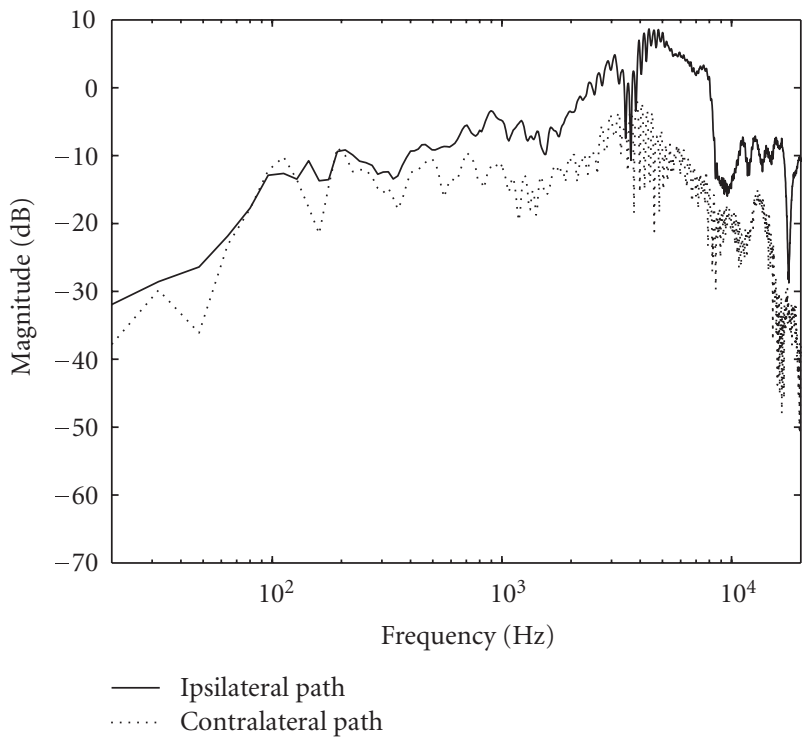

FIGURE 3: The frequency responses of the plants including ipsilateral and contralateral paths.

bandlimited CCS is 1500. In other words, the frequency (under $6 \mathrm{kHz}$ ) resolution of the bandlimited CCS is twice than that of the fullband CCS. That is, the bandlimited CCS has finer resolution. Figure 7 (a) shows the frequency responses of $Q_{11 b}$ and $Q_{12 b}$, where the subscript $b$ stands for the bandlimited method, represented as solid line and dotted line, respectively. The channel separation, $Q_{12 b} / Q_{11 b}$, is shown in Figure 7(b) as the dotted line. From Figures 4(b) and 7(b), we can see that the bandlimited CCS gets better channel separation, especially from $100 \mathrm{~Hz}$ to $1 \mathrm{kHz}$.

Subjective listening experiment includes two parts: the source localization test and the sound quality test. Eleven subjects participated in the test. The listeners were instructed to sit at the position where KEMAR was. In the first part, the test stimulus was a pink noise bandlimited to $20 \mathrm{kHz}$. Each stimulus was played 5 times in $25 \mathrm{~ms}$ duration with $50 \mathrm{~ms}$ silent interval. Virtual sound images at 7 prespecified directions on the right horizontal plane with increment $30^{\circ}$ azimuth are rendered by using HRTFs. Listeners were 


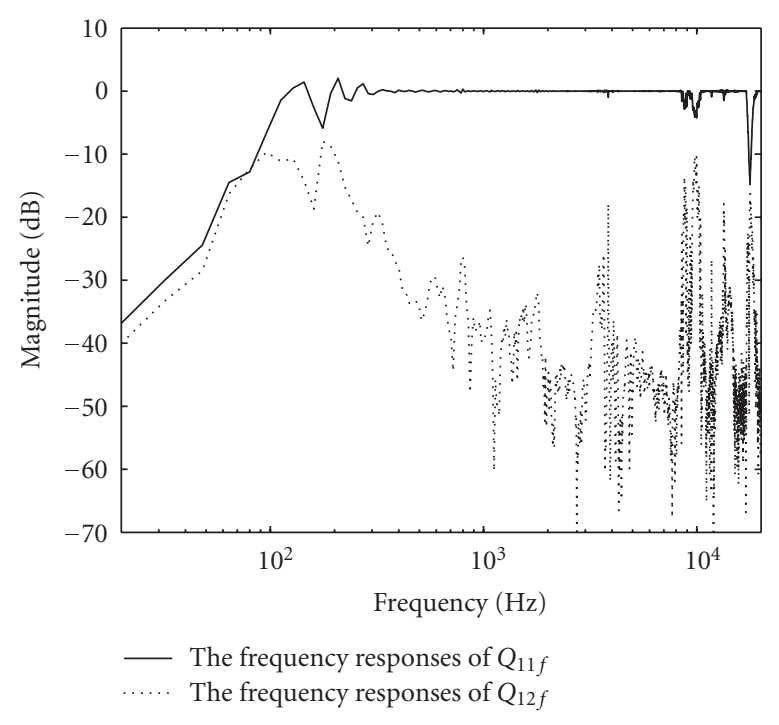

(a)

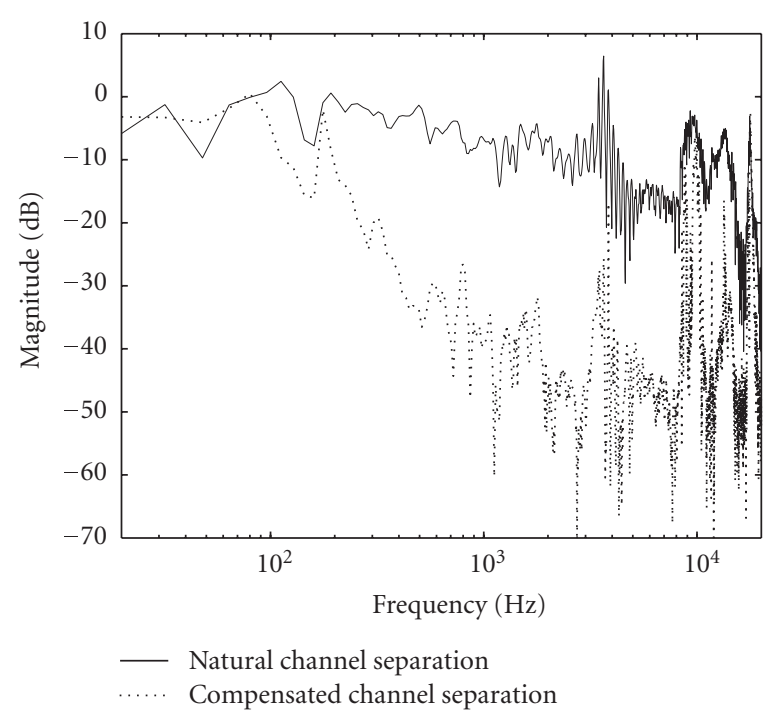

(b)

FIgURE 4: (a) The frequency responses of $Q_{11 f}$ and $Q_{12 f}$. (b) Natural channel separation and compensated channel separation.

well trained by playing the stimuli of all angles prior to the test. The experiments were blind tests in which stimuli were played randomly without informing the subjects the source direction. The results of localization test are shown in terms of target angles versus judged angles in Figures 8(a) and 8(b), corresponding to the cases of fullband CCS and bandlimited CCS. The size of each circle is proportional to the number of the listeners who localized the same perceived angle. The 45degree line indicates the perfect localization. It is observed from the results that subjects localized well at front $(0 \mathrm{de}-$ gree) and back (180 degrees) no matter what approach is employed. While the fullband CCS performs well at 30-degree angle, subjects were confused within the range $60^{\circ}-120^{\circ}$. On the other hand, bandlimited CCS performs slightly better

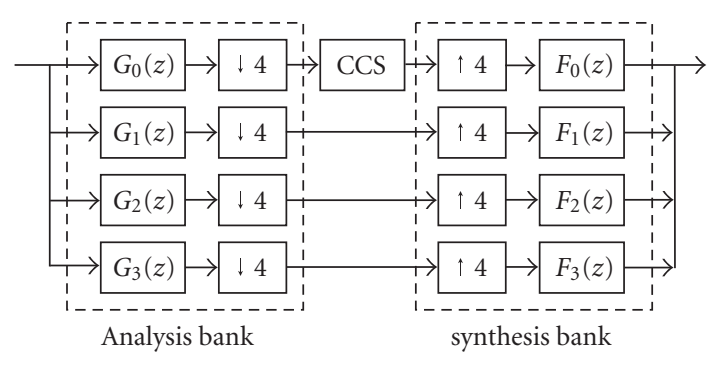

FIGURE 5: The block diagram of the bandlimited CCS.

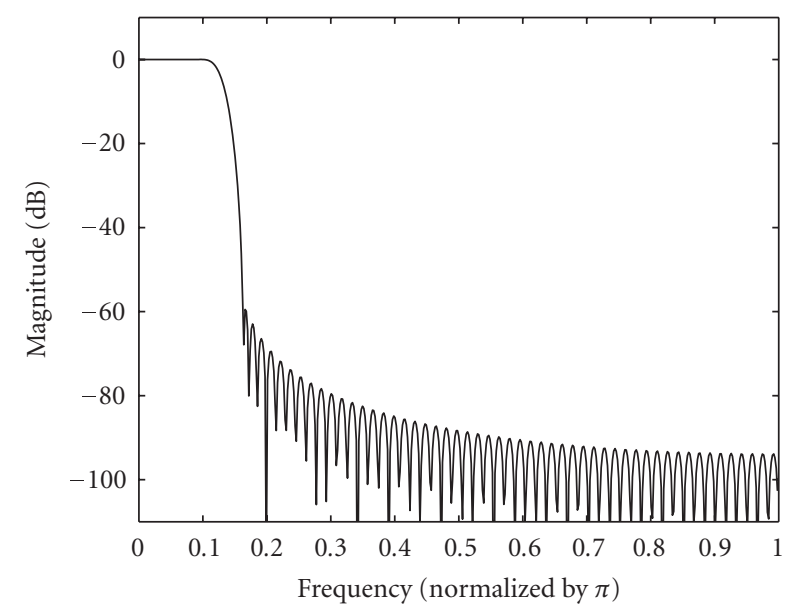

(a)

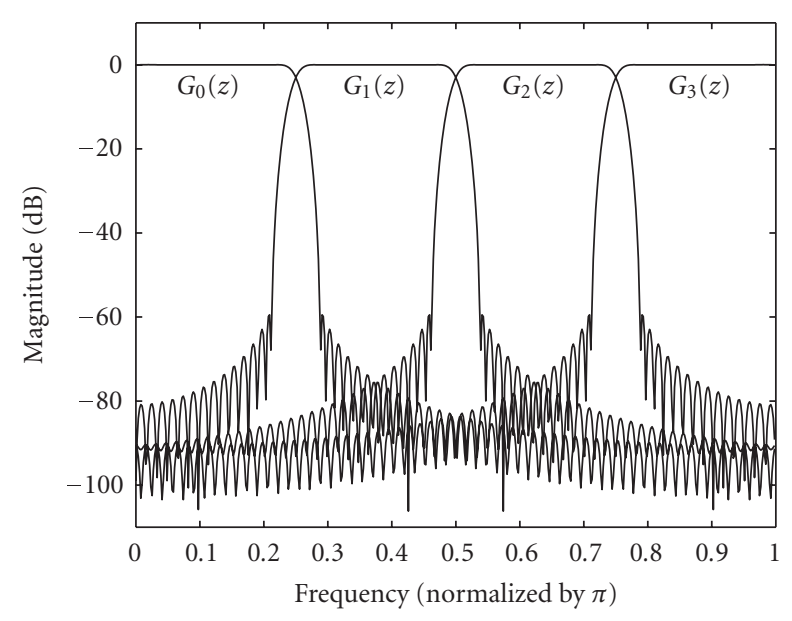

(b)

FIGURE 6: The magnitude responses of (a) prototype FIR filter and (b) analysis bank.

within the range $60^{\circ}-120^{\circ}$. It is interesting to note that bandlimited CCS exists no back-front reversal problem which means that the subject localizes rear stimulus to front angle. In addition, a one-way analysis of variance (ANOVA) on the subjective localization result was conducted. These results were preprocessed into five levels of grade, as described in Table 1. Figure 9(a) shows the means and spreads (with 


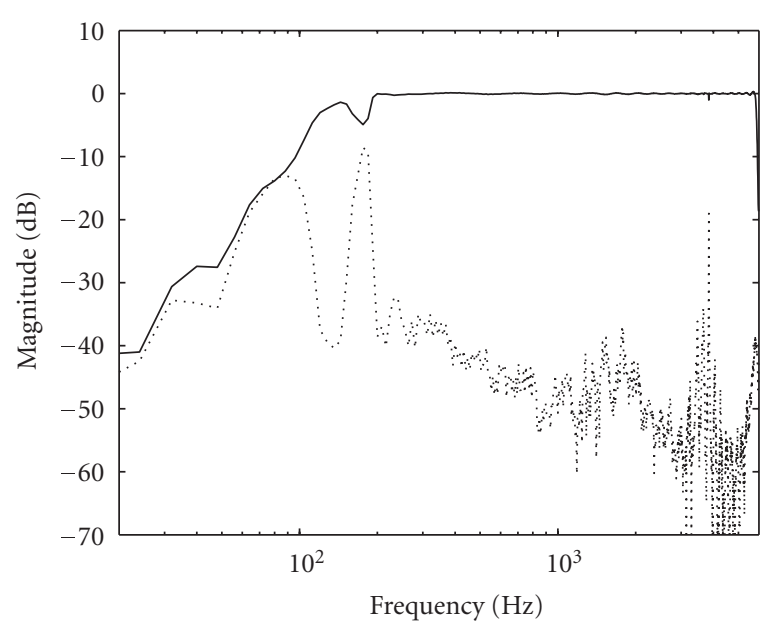

- The frequency responses of $Q_{11 b}$ The frequency responses of $Q_{12 b}$

(a)

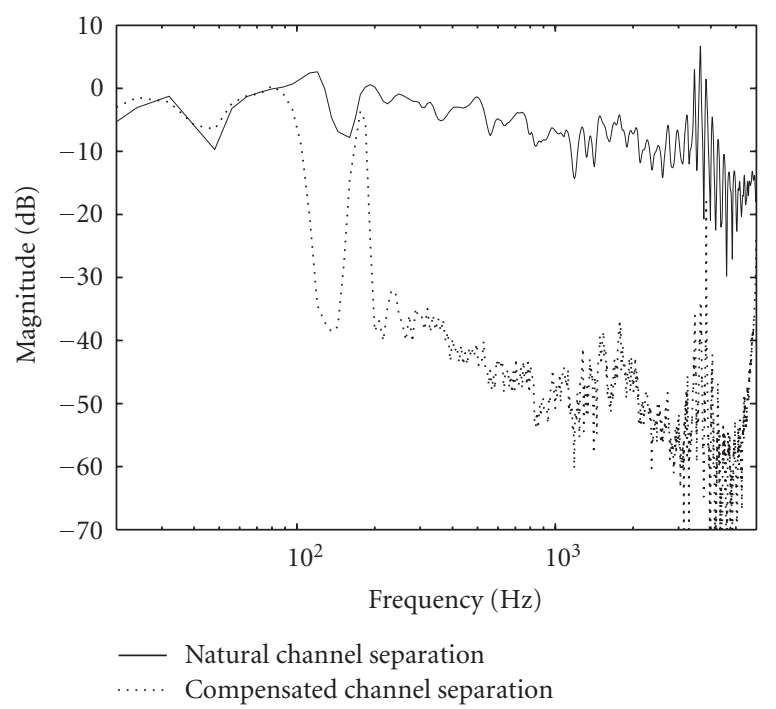

(b)

FIgURE 7: (a) The frequency responses of $Q_{11 b}$ and $Q_{12 b}$. (b) Natural channel separation and compensated channel separation.

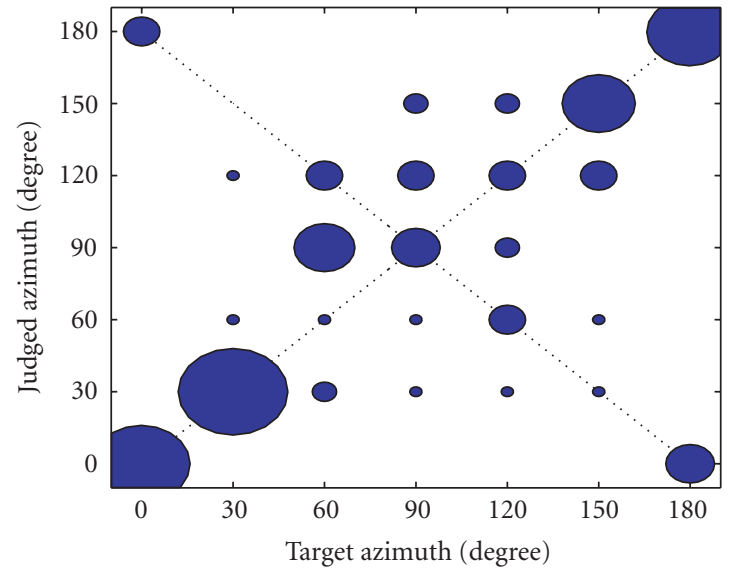

(a)

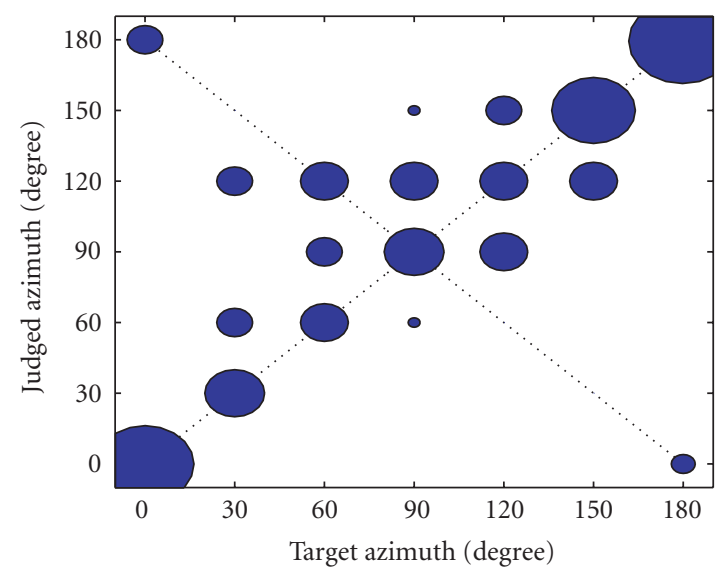

(b)

FIgURE 8: Results of the subjective localization test of azimuth. (a) Fullband CCS. (b) Bandlimited CCS.

95\% confidence intervals) of the grades for two kinds of approaches. The mean of the bandlimited CCS is slightly larger than that of the fullband CCS as we observed previously. ANOVA output reveals that two approaches are not statistically significant $(p=0.2324>0.05)$.

In the second part, the stimulus prefiltered by the fullband CCS and the bandlimited CCS were treated as the reference and the object, respectively. The "double-blind triple stimulus with hidden reference" method has been employed in this testing procedure [25]. A listener at a time was involved in three stimuli (" $A$," " $B$," and " $C$ ") where " $A$ " represented the reference and " $B$ " and/or " $C$ " represented the hidden reference and/or the object. A subject was requested to compare " $B$ " to " $A$ " and " $C$ " to " $A$ " with five-grade impair- ment scale described in Table 2 . The test stimuli contain three types of music including a bass (low frequency), a triangle (high frequency), and a popular song (comprehensive effect). Figure 9(b) shows the means and spreads (with 95\% confidence intervals) of the grades for two kinds of approaches. It seems that the fullband CCS earned a slightly higher grade than the subband approach since the fullband CCS was used as the reference. Nevertheless, ANOVA test reveals that the performance difference between two approaches is not statistically significant $(p=0.4109>0.05)$.

Here, the proposed method has been validated that it performs comparably well as the fullband CCS. In Table 3, two approaches are compared in terms of computation loading, where MPU and APU represent multiplications and 
TABLE 1: Description of five levels of grade for the subjective localization test.

\begin{tabular}{l|c}
\hline Description & Grade \\
\hline The judged angle is the same as the target angle & 5.0 \\
$30^{\circ}$ difference between the judged angle and the target angle & 4.0 \\
Front-back reversal of the judged angle identical to the target angle & 3.0 \\
$30^{\circ}$ difference between front-back reversal of the judged angle and the target angle & 2.0 \\
Otherwise & 1.0 \\
\hline
\end{tabular}

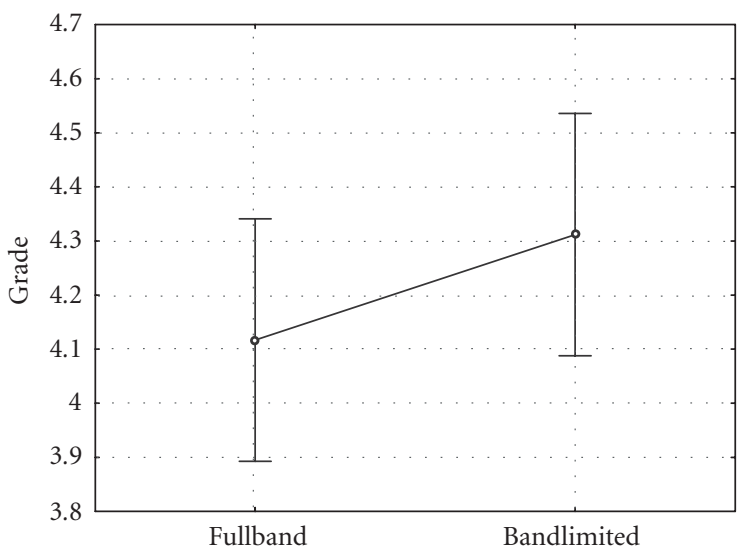

(a)

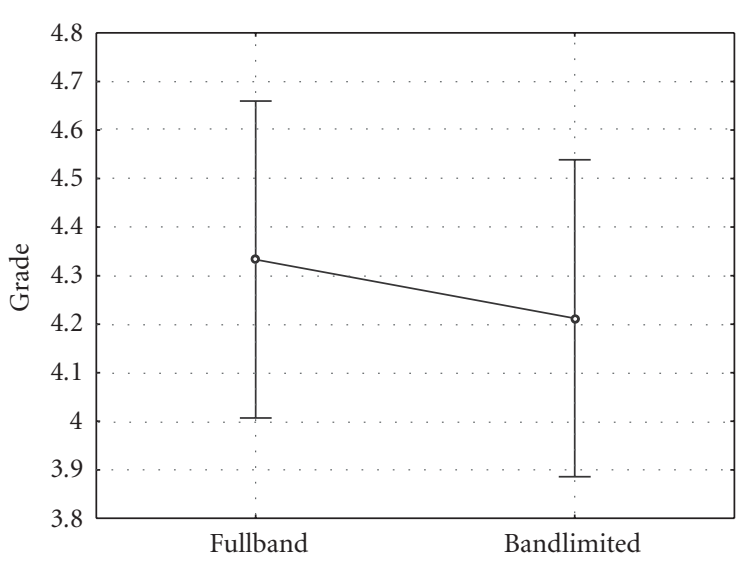

(b)

Figure 9: Means and spreads (with 95\% confidence intervals) of the grades for two kinds of CCS approaches. (a) Grades of the source localization experiment. (b) Grades of the sound quality tests.

additions per unit time, respectively. The computation loadings are calculated using direct convolution in the time domain. The computation loading using the proposed subband filtering approach was drastically reduced by approximately eighty percent, as compared to the conventional approach. However, there are still other fast convolution algorithms that can be adopted for efficient implementation. The overlap-add methods of block convolution [26], for example, are compared in the simulation. This method is only used in CCS filters, while the filter bank is still carried out by using
TABLE 2: Five-grade impairment scale.

\begin{tabular}{l|c}
\hline Impairment & Grade \\
\hline Imperceptible & 5.0 \\
Perceptible, but not annoying & 4.0 \\
Slightly annoying & 3.0 \\
Annoying & 2.0 \\
Very annoying & 1.0 \\
\hline
\end{tabular}

TABLE 3: The comparison of computation loading of the fullband CCS and the bandlimited CCS with direct convolution.

\begin{tabular}{l|cc}
\hline & Fullband & Bandlimited \\
\hline MPU & 12000 & 1980 \\
APU & 11998 & 1976 \\
\hline
\end{tabular}

TABLE 4: The comparison of computation loading of the fullband CCS and the bandlimited CCS with fast convolution.

\begin{tabular}{l|cc}
\hline & Fullband & Bandlimited \\
\hline MPU & 1464 & 815 \\
APU & 1462 & 808 \\
\hline
\end{tabular}

direct convolution because of the efficient polyphase implementation. In the procedure of block convolution, the fast Fourier transform is used to realize discrete Fourier transform. Moreover, the number of complex multiplications and additions of the fast Fourier transform is equal to $\mathrm{N} \log _{2} \mathrm{~N}$, where $N$ is the number of the transform point. After using block convolution, the results of computation loading are listed in Table 4.

The shuffler method can be applied due to symmetric assumption. The shuffler structure is shown in Figure 10. It saves around fifty percent of computation [19]. The multichannel shuffler structure can be found in [18].

\section{CONCLUSIONS}

A bandlimited CCS based on subband filtering has been developed in the work. The intention is to establish a computationally efficient CCS without penalty on cancellation performance. The CCS is a bandlimited design which is effective up to the frequency $6 \mathrm{kHz}$. To achieve the bandlimited implementation, a pseudocosine modulated QMF is employed, allowing the CCS to operate at low rate within an approximate 


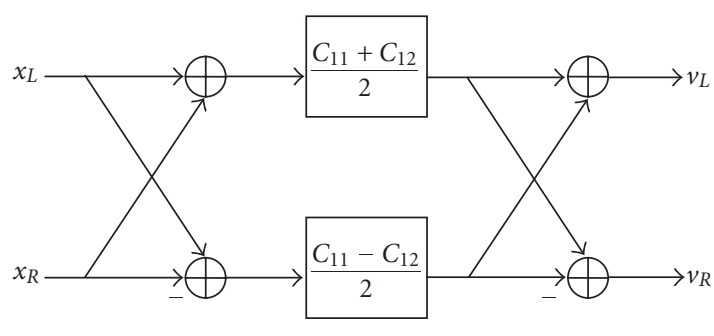

FIGURE 10: Shuffler filter structure for $2 \times 2$ CCS.

PR structure. As a result of this, spatial audio processing can concentrate more on the low frequency range to better suit human perceptual hearing.

To compare the proposed CCS to traditional systems, subjective listening experiments were conducted in an anechoic room. The experiments include two parts: source localization test and sound quality test. By means of the techniques presented in Section 2, the fullband CCS operated at the sampling rate of $48 \mathrm{kHz}$ requires four 3000-tapped FIR filters. On the other hand, the bandlimited CCS operated at the sampling rate of $12 \mathrm{kHz}$ requires only four 1500-tapped FIR filters. The prototype FIR filter has 120 taps. The analysis bank and the synthesis bank are generated from the prototype and implemented via polyphase representation. The results of subjective tests processed by ANOVA indicate that the bandlimited CCS performs comparably well as the fullband CCS not only in localization but also in sound quality. From Table 3, the computation loading using the proposed subband filtering approach was drastically reduced by approximately eighty percent, as compared to the conventional approach. After employing fast convolution algorithm, the difference between two methods is reduced. Even though the block convolution is very efficient, it requires more memory to store temporary data. In conclusion, which method is better is dependent upon which one you concern about, speed or memory. The bandlimited CCS with direct convolution and shuffler method is an acceptable choice.

\section{ACKNOWLEDGMENT}

The work was supported by the National Science Council in Taiwan, under project number NSC94-2212-E009-019.

\section{REFERENCES}

[1] J. Blauert, Spatial Hearing: The Psychophysics of Human Sound Localization, MIT Press, Cambridge, Mass, USA, 1997.

[2] D. R. Begault, 3-D Sound for Virtual Reality and Multimedia, AP Professional, Cambridge, Mass, USA, 1994.

[3] A. Sibbald, "Transaural acoustic crosstalk cancellation," Sensaura White Papers, 1999, http://www.sensaura.co.uk.

[4] M. R. Schroeder and B. S. Atal, "Computer simulation of sound transmission in rooms," IEEE International Convention Record, vol. 11, no. 7, pp. 150-155, 1963.

[5] P. Damaske and V. Mellert, "A procedure for generating directionally accurate sound images in the upper- half space using two loudspeakers," Acoustica, vol. 22, pp. 154-162, 1969.
[6] D. H. Cooper, "Calculator program for head-related transfer function," Journal of the Audio Engineering Society, vol. 30, no. 1-2, pp. 34-38, 1982.

[7] W. G. Gardner, "Transaural 3D audio," Tech. Rep. 342, MIT Media Laboratory, Cambridge, Mass, USA, 1995.

[8] D. H. Cooper and J. L. Bauck, "Prospects for transaural recording," Journal of the Audio Engineering Society, vol. 37, no. 1-2, pp. 3-19, 1989.

[9] J. L. Bauck and D. H. Cooper, "Generalized transaural stereo and applications," Journal of the Audio Engineering Society, vol. 44, no. 9, pp. 683-705, 1996.

[10] O. Kirkeby and P. A. Nelson, "Digital filter design for inversion problems in sound reproduction," Journal of the Audio Engineering Society, vol. 47, no. 7, pp. 583-595, 1999.

[11] O. Kirkeby, P. A. Nelson, H. Hamada, and F. OrdunaBustamante, "Fast deconvolution of multichannel systems using regularization," IEEE Transactions on Speech and Audio Processing, vol. 6, no. 2, pp. 189-194, 1998.

[12] C. Kyriakakis, T. Holman, J.-S. Lim, H. Hong, and H. Neven, "Signal processing, acoustics, and psychoacoustics for high quality desktop audio," Journal of Visual Communication and Image Representation, vol. 9, no. 1, pp. 51-61, 1998.

[13] C. Kyriakakis, "Fundamental and technological limitations of immersive audio systems," Proceedings of the IEEE, vol. 86, no. 5, pp. 941-951, 1998.

[14] O. Kirkeby, P. A. Nelson, and H. Hamada, "The "stereo dipole" - a virtual source imaging system using two closely spaced loudspeakers," Journal of the Audio Engineering Society, vol. 46, no. 5, pp. 387-395, 1998.

[15] M. R. Bai, C.-W. Tung, and C.-C. Lee, "Optimal design of loudspeaker arrays for robust cross-talk cancellation using the Taguchi method and the genetic algorithm," Journal of the Acoustical Society of America, vol. 117, no. 5, pp. 2802-2813, 2005.

[16] T. Takeuchi, P. A. Nelson, and H. Hamada, "Robustness to head misalignment of virtual sound imaging systems," Journal of the Acoustical Society of America, vol. 109, no. 3, pp. 958971, 2001.

[17] J. Yang, W.-S. Gan, and S.-E. Tan, "Improved sound separation using three loudspeakers," Acoustic Research Letters Online, vol. 4, no. 2, pp. 47-52, 2003.

[18] J. Yang, W.-S. Gan, and S.-E. Tang, "Development of virtual sound imaging system using triple elevated speakers," IEEE Transactions on Consumer Electronics, vol. 50, no. 3, pp. 916922, 2004.

[19] W. G. Gardner, 3-D Audio Using Loudspeakers, Kluwer Academic, London, UK, 1998.

[20] P. P. Vaidyanathan, Multirate Systems and Filter Banks, Prentice-Hall, Englewood Cliffs, NJ, USA, 1993.

[21] M. R. Bai and C.-C. Lee, "Development and implementation of cross-talk cancellation system in spatial audio reproduction based on subband filtering," Journal of Sound and Vibration, vol. 290, no. 3-5, pp. 1269-1289, 2006.

[22] B. Noble, Applied Linear Algebra, Prentice-Hall, Englewood Cliffs, NJ, USA, 1988.

[23] A. Schuhmacher, J. Hald, K. B. Rasmussen, and P. C. Hansen, "Sound source reconstruction using inverse boundary element calculations," Journal of the Acoustical Society of America, vol. 113, no. 1, pp. 114-127, 2003.

[24] Y.-P. Lin and P. P. Vaidyanathan, "A Kaiser window approach for the design of prototype filters of cosine modulated filterbanks," IEEE Signal Processing Letters, vol. 5, no. 6, pp. 132134, 1998. 
[25] Rec. ITU-R BS.1116-1, "Method for the subjective assessment of small impairments in audio systems including multichannel sound systems," International Telecommunications Union, Geneva, Switzerland, 1992-1994.

[26] A. V. Oppenheim, R. W. Schafer, and J. R. Buck, Discrete-Time Signal Processing, Prentice-Hall, Upper Saddle River, NJ, USA, 2nd edition, 1999.

Mingsian R. Bai was born in 1959 in Taipei, Taiwan. He received the Bachelor's degree in power mechanical engineering from $\mathrm{Na}-$ tional Tsing-Hwa University in 1981. He also received the Master's degree in business management from National Chen-Chi University in 1984. He left Taiwan in 1984 to enter graduate school of Iowa State University and later received the M.S. degree in mechanical engineering in 1985 and the

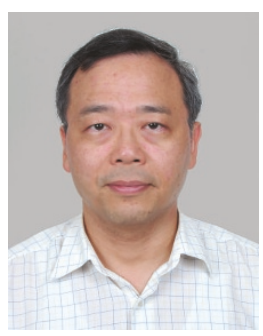
Ph.D. degree in engineering mechanics and aerospace engineering in 1989. In 1989, he joined the Department of Mechanical Engineering of National Chiao-Tung University in Taiwan as an Associate Professor and became a Professor in 1996. He was also a Visiting Scholar to Center of Vibration and Acoustics, Penn State University, University of Adelaide, Australia, and Institute of Sound and Vibration Research (ISVR), UK, in 1997, 2000, and 2002, respectively. His current interests encompass acoustics, audio signal processing, electroacoustic transducers, vibroacoustic diagnostics, active noise and vibration control, and so forth. He has over 100 published papers and 13 granted or pending patents. He is a Member of the Audio Engineering Society (AES), Acoustical Society of America (ASA), Acoustical Society of Taiwan, and Vibration and Noise Control Engineering Society in Taiwan.

Chih-Chung Lee was born in 1979 in Taipei, Taiwan. He received the B.S. degree and the M.S. degree in mechanical engineering from National Chiao-Tung University in 2001 and 2003, respectively. His Master's thesis is on personal $3 \mathrm{D}$ virtual cinema based on panel speaker array. He is currently studying the Ph.D. degree in mechanical engineering from National Chiao-Tung

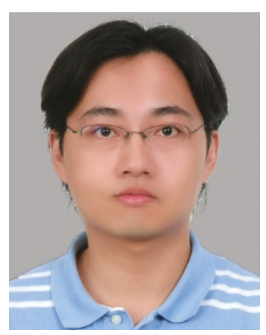
University. 